ISSN 0103-9954

\title{
COMPARAÇÃO DE DIFERENTES MÉTODOS DE AMOSTRAGEM, DE ÁREA FIXA E VARIÁVEL, EM UMA FLORESTA DE Araucaria angustifolia
}

\section{COMPARASION OF DIFFERENT SAMPLING METHODS, OF FIXED AND VARIABLE AREAS, IN AN Araucaria angustifolia FOREST}

\author{
Fabio Abel Moscovich ${ }^{1}$ Doádi Antônio Brena ${ }^{2}$ Solon Jonas Longhi ${ }^{3}$
}

\section{RESUMO}

Este trabalho foi desenvolvido em uma Floresta de Araucaria angustifolia pertencente à Floresta Nacional de São Francisco de Paula - RS. O objetivo foi comparar quatro métodos de amostragem de área variável (Strand, Prodan, Quadrantes e Bitterlich) com o método de Área Fixa e determinar a eficiência de cada um deles nas estimativas dos parâmetros quantitativos (volume comercial com casca, número de árvores e área basal) e qualitativos (abrangência das espécies) da população. Realizou-se o inventário de um hectare considerando todas as árvores com circunferência altura do peito (CAP) $30 \mathrm{~cm}$. Estas foram identificadas e medidas suas alturas totais e comerciais. $\mathrm{O}$ método de Área Fixa adotado foi o de faixas com $100 \mathrm{~m}$ de comprimento e $10 \mathrm{~m}$ de largura (10 faixas contíguas). Para os métodos de Prodan, Quadrantes e Bitterlich foram realizados 25 pontos amostrais distribuídos sistematicamente a cada $20 \mathrm{~m}$ e do método de Strand realizaram-se 30 linhas de amostragem de 15,7 m de comprimento. Pela análise de variância, verificou-se que os distintos métodos não apresentaram diferenças significativas na estimativa do volume, área basal e número de árvores por hectare. Encontrou-se diferença significativa no número de espécies amostradas, sendo o método de Área Fixa o que apresentou maior média. Com os tempos tomados e os coeficientes de variação encontrados para cada estimativa foi calculada a eficiência relativa, na qual verificouse que, o método de Strand foi sempre superior aos demais métodos na estimação de todos os parâmetros. Em contraposição, o método dos Quadrantes foi o que apresentou a menor eficiência relativa na estimação de todos os parâmetros.

Palavras-chave: Métodos de Amostragem, Floresta de Araucaria angustifolia, Inventário Florestal.

\begin{abstract}
This research was carried out in an Araucaria angustifolia forest which belongs to The National Forest of São Francisco de Paula, RS state. Its purpose was to compare four sampling methods of variable areas (Strand, Prodan, Quadrant and Bitterlich) with the method of Fixed Area Plot; and to

1 Engenheiro Florestal, Msc., Acadêmico do Curso de Pós-Graduação (Doutorado) em Engenharia Florestal, Centro de Ciências Rurais, Universidade Federal de Santa Maria, Av. Roraima, 1000, CEP: 97105-900, Santa Maria - RS.

2 Engenheiro Florestal, Dr., Professor Adjunto do Departamento de Ciências Florestais, Centro de Ciências Rurais, Universidade Federal de Santa Maria, Av. Roraima, 1000, CEP: 97105-900, Santa Maria - RS.

3 Engenheiro Florestal, Dr., Professor Titular do Departamento de Ciências Florestais, Centro de Ciências Rurais, Universidade Federal de Santa Maria, Av. Roraima, 1000, CEP: 97105-900, Santa Maria - RS.
\end{abstract}


Moscovich, F. A.; Brena, D. A.; Longhi, S. J.

determine the efficiency of each method in the estimation of quantitative parameters (CommerciaI out bark volume, number of trees and basal area), and the qualitative ones (ocurring species) of the population. The inventory of a hectare tract of forest was done considering all the trees with a breast height circumference (CAP) $30 \mathrm{~cm}$. These trees were identified and their total commercial heights were measured. The Fixed Area Method adopted was the one with strips of $100 \mathrm{~m}$ of length and $10 \mathrm{~m}$ of width ( 10 contiguous strips of land). For the Prodan, Quadrant and Bitterlich methods, 25 points were established systematically distributed along $20 \mathrm{~m}$ and in the Strand Method 30 contiguous lines of $15,7 \mathrm{~m}$ of length. Through analysis of variance, it was observed that these methods did not show significant differences in term of volume estimation, basal area and number of trees. A significant difference was found for the number of ocurring species, being the Fixed Area Method the one that showed the highest average, differing from the other methods. After writing down the time and finding the coefficient of variation for each estimation, the relative efficiency, the result was that the Strand Method was always superior to the others in estimation of all parameters. On the other hand, the Quadrant Method showed the lowest relative efficiency in the parameter estimation.

Key words: Sampling Methods, Araucaria angustifolia Forest, Forest Inventory.

\section{INTRODUÇÃO}

As estimativas dos parâmetros de uma população são obtidas por meio da medição de características de um conjunto de unidades de amostra tomado como representativo dessa população. As unidades de amostra são constituídas de parcelas de tamanho fixo ou variável, variando, em razão disso, os métodos de amostragem a serem empregados.

A amostragem por parcelas de tamanho fixo é a mais utilizada nos inventários florestais, sendo a probabilidade de seleção das árvores, proporcional à área da parcela e à frequência dos indivíduos que nela ocorrem (PÉLLICO NETTO \& BRENA, 1997).

Nas parcelas de área variável, a probabilidade de seleção é proporcional à área basal ou distância das árvores a um ponto ou linha. Sabe-se que a amostragem por parcelas, ou por pontos, ou linhas, é uma técnica apropriada para seleção de árvores, por processo casual ou sistemático, fornecendo estimativas dignas de confiança de alguns parâmetros da população, como volume, número de árvores, área basal, entre outros (MYERS \& BEERS apud SOUZA et al., 1981).

O tamanho e a forma da parcela de Área Fixa, bem como o método de amostragem de Área Variável a ser empregados, irão influenciar a eficiência de um inventário florestal, refletido pelo nível de precisão, e pela exatidão dos valores estimados, bem como pelo custo do levantamento.

O objetivo geral do presente trabalho foi comparar os métodos de amostragem de Strand, de Prodan, de Bitterlich e de Quadrantes com o método de Área Fixa, aplicados a uma Floresta Ombrófila Mista.

Os objetivos específicos foram:

a) comparar a precisão das estimativas quantitativas nos métodos de Strand, Prodan, Quadrantes e Bitterlich com o método de Área Fixa;

b) comparar os tempos de coleta de dados demandados pelo método de Strand, Prodan, Quadrantes

Ci. Fl., v. 1, n. 1, 1991 
e Bitterlich com o método de Área Fixa;

c) comparar a abrangência de espécies florestais estimada pelos métodos de Strand, Prodan, Bitterlich e Quadrantes com o método de Área Fixa.

\section{REVISÃO DA LITERATURA}

\section{Método de Área Fixa}

Neste método de amostragem a seleção dos indivíduos é feita proporcional à área da unidade e, consequentemente, à frequência dos indivíduos que nela ocorrem. Este é o mais antigo e conhecido método de amostragem. As variações da forma e tamanho das unidades amostrais constituem as variáveis fundamentais para avaliação de sua aplicação prática (PÉLLICO NETTO \& BRENA, 1997). A forma e o tamanho das unidades amostrais têm sido decididas muito mais pela praticidade e operacionalidade de sua localização e demarcação em campo, do que por qualquer outra argumentação.

Segundo Pearce apud Péllico Netto \& Brena (1997), pode-se afirmar que não há informações acerca do melhor tamanho para unidades amostrais, mas observa que as pequenas proporcionam economia de tempo, enquanto as maiores proporcionam redução de mão de obra.

Devido à grande variação de tipologias e espécies que ocorrem nas florestas naturais do país, as unidades retangulares têm sido preferidas. Unidades com até $250 \mathrm{~m}$ de comprimento têm permitido detectar a variação de espécies, normalmente ocorrentes em comunidades ou unidades gregárias, dentro das diferentes tipologias (PÉLLICO NETTO \& BRENA, 1997).

Como se pode observar, não há uma consistência na decisão sobre o tamanho dessas unidades e resta, na opinião dos pesquisadores, que este tamanho seja decidido à base de experiência prática e de um confronto entre precisão e custos.

No que se refere à forma das unidades amostrais, Pearce apud Péllico Netto \& Brena (1997) diz também, que as unidades amostrais estreitas e compridas, de maneira geral, são melhores que as quadradas, porém, muitas vezes, as quadradas se sobrepõem àquelas e a decisão sobre uma ou outra forma depende do propósito do estudo.

Segundo Robles (1978), em termos gerais, pode-se afirmar que unidades amostrais compridas e estreitas são mais convenientes do ponto de vista da desuniformidade do solo. Com a forma comprida é mais factível que as "vetas" ou "manchas" de fertilidade sejam alcançadas por igual nas diferentes unidades amostrais, diminuindo assim as diferenças entre as unidades.

\section{Método de Strand}

O Método da Linha ou Método de Strand é uma aplicação de técnica de contagem angular (LOETSCH et al., 1973).

Strand apud Péllico Netto \& Brena (1997) focaliza neste método fundamentalmente o critério probabilístico de seleção dos indivíduos na unidade amostral com proporcionalidade ao diâmetro, para o cálculo da área basal e número de árvores por hectare, e proporcionalidade à altura das 
Moscovich, F. A.; Brena, D. A.; Longhi, S. J.

árvores, para se obter o volume e também o número de árvores por hectare. Sua abordagem é feita em linhas dentro da floresta e não em pontos de estação como no caso de Bitterlich, Prodan e Quadrantes.

A unidade amostral é constituída de uma linha de comprimento (Lz5rt), tomada dentro do povoamento, sobre a qual enumeram-se todas as árvores do seu lado esquerdo que se qualificam para amostragem. A amostragem é realizada em duas etapas:

a) $\mathrm{Na}$ primeira etapa, a seleção das árvores a serem medidas é feita com probabilidade proporcional ao diâmetro, através do método de Bitterlich, observando-se apenas as árvores situadas à esquerda de linha de comprimento (L), para estimar a área basal e o número de árvores por hectare. Em cada árvore selecionada mede-se o diâmetro a altura do peito (DAP).

b) Na segunda etapa de amostragem, volta-se a caminhar pela linha e selecionam-se as árvores a serem medidas com probabilidade proporcional à altura, para estimar o volume por hectare. Enumeram-se as árvores situadas à sua esquerda, cuja distância da árvore à linha seja igual ou menor que a metade da sua altura total, ou seja, $(\mathrm{D} \leq \mathrm{h} / 2)$ (PÉLLICO NETTO \& BRENA, 1997).

\section{Método de Prodan}

Este método foi primeiramente apresentado por Prodan em 1968 em Freiburg - Alemanha. Este considera a medição de 6 árvores e a distância ou raio do centro do ponto amostral ao centro da sexta árvore como referência da unidade amostral. Nestas condições, a inclusão de uma árvore na amostragem tem como variável a sua distância ao ponto amostral e, portanto, a seleção das árvores se faz com probabilidade proporcional à distância (PÉLLICO NETTO \& BRENA, 1997).

A decisão sobre o número de árvores medidas na unidade amostral, teve como base o comportamento do coeficiente de variação, do volume individual das árvores, em relação ao número de árvores observadas. Neste estudo, Prodan (1968) verificou que o coeficiente de variação se tornava estável a partir da sexta árvore medida. (PÉLLICO NETTO \& BRENA, 1997).

A unidade amostral consiste em se medirem as seis árvores mais próximas de um ponto amostral. A sexta árvore é contada como meia árvore.

\section{Método dos Quadrantes}

Segundo Martins (1993), o Método dos Quadrantes teve origem nos levantamentos realizados pelos agrimensores do United States Land Survery Service, no Estado de Wisconsin, Estados Unidos, durante os anos 1833 a 1834.

Este método pertence à classe dos métodos de distâncias, uma vez que a probabilidade de seleção dos indivíduos a serem amostrados em cada quadrante é proporcional a sua distância ao centro da unidade (MARTINS, 1993).

A unidade amostral é definida por um ponto. Cada ponto representa o centro de quatro quadrantes. Dentro de cada quadrante, seleciona-se a árvore mais próxima ao ponto, mede-se a distância radial (Q) do ponto ao centro da árvore, seu diâmetro, sua altura e registra-se sua

Ci. Fl., v. 1, n. 1, 1991 
identificação botânica (BRENA, 1996).

\section{Método de Bitterlich}

Os estudos de telescopia tiveram início com Bitterlich em 1931, sendo a primeira publicação de 1947 (FINGER, 1992; LOETSCH et al., 1973; HUSCH et al., 1982).

O método baseia-se no postulado de Bitterlich que (FINGER, 1992) "O número de árvores de um povoamento, cujos diâmetros a altura do peito (DAPs) a partir de um ponto fixo aparecem superior a um valor angular alfa constante, é proporcional à área basal em metros quadrados por hectare".

O método consiste em contar as árvores, em um giro de $360^{\circ}$, cujos diâmetros forem iguais ou maiores que a abertura angular equivalente a, em que () é um ângulo fixo, cujo vértice é o ponto central da unidade amostral. A seleção das árvores é, portanto, efetuada com probabilidade proporcional à área basal, ou ao quadrado do diâmetro e à frequência (PÉLLICO NETTO \& BRENA,1997).

A amostra de contagem angular resulta da determinação do número de árvores de um povoamento, cujo DAP, a partir de um ponto fixo, aparece superior a um dado valor angular constante. Estes valores constituem a medida básica para determinar a área basal (HUSCH et al., 1982; PÉLLICO NETTO \& BRENA, 1997; FINGER, 1992; LOETSCH et al., 1973).

Em cada árvore selecionada nos pontos de Bitterlich mede-se o diâmetro à altura do peito, a altura e identifica-se a espécie.

\section{MATERIAIS E MÉTODOS}

O estudo foi desenvolvido em uma unidade amostral permanente de 1 hectare, instalada em 1995 na Floresta Nacional (FLONA) de São Francisco de Paula, na região fisiográfica chamado de Planalto das Araucárias pelo projeto RADAMBRASIL (FONTANA, 1994), na Encosta Superior do Nordeste do Rio Grande do Sul (BRASIL, 1973). A vegetação natural da FLONA de São Francisco de Paula pertence ao tipo fitogeográfico "Mata de Araucária ou Pinheiro Brasileiro" (IBDF/FATEC, 1989; RAMBO, 1956; HUECK, 1972; KLEIN, 1960; REITZ \& KLEIN, 1966; LINDMAN \& FERRI, 1974), ou "Floresta Ombrófila Mista" (VELOSO \& GÓES FILHO, 1982).

Os métodos de amostragem (Área Fixa, Strand, Prodan, Quadrantes e Bitterlich) foram aplicados sobre a parcela permanente, de modo a cobri-la com o número potencial de unidades amostrais de cada método, evitando-se qualquer sobreposição de unidade. Desse modo, a parcela permanente foi coberta por 10 unidades do método de Área Fixa; 25 unidades dos métodos de Bitterlich, Prodan e Quadrantes; e 30 unidades do método de Strand.

\section{Método de Área Fixa}

O método de Área Fixa foi constituído de 10 faixas com $100 \mathrm{~m}$ de comprimento por $10 \mathrm{~m}$ de largura. Este método foi tomado como padrão para a comparação dos parâmetros obtidos pelos 
demais métodos em estudo.

As medições foram realizadas a partir da primeira faixa, registrando-se o tempo desde o início até a conclusão da coleta de dados. As árvores de cada faixa foram identificadas, medidas as circunferências a altura do peito (CAP) com trena, com aproximação de centímetro, e as alturas total e comercial com um hipsômetro Blume-Leiss, com precisão de 0,5 m.

A conversão das estimativas amostrais para hectare, foi feita utilizando-se o fator de probabilidade, proposto por Péllico Netto \& Brena (1997), calculado da seguinte maneira:

Em que:

$$
\mathrm{F}=\mathrm{A} / \mathrm{a}
$$

$\mathrm{A}=$ área de um hectare;

$\mathrm{a}=$ área da unidade amostral.

As estimativas para a área basal, número de árvores e volume, foram obtidas pelas seguintes equações:

a) Estimativa da área basal por hectare

A área basal por hectare $(G)$ foi obtida pelo somatório das áreas de seção transversal $\left(g_{\mathrm{j}}\right)$ das (m) árvores que ocorreram na unidade amostral e multiplicado pelo fator de proporcionalidade $(\mathrm{F})$.

$$
G=\left(\sum_{i=1}^{m} g_{i}\right) F
$$

b) Estimativa do número de árvores por hectare

O número de árvores por hectare $(N)$ foi obtido multiplicando-se o número de árvores medido na unidade $(m)$ pelo fator de proporcionalidade $(\mathrm{F})$.

$$
\mathrm{N}=\mathrm{mF}
$$

c) Estimativa do volume comercial com casca por hectare

$\mathrm{O}$ volume comercial por hectare $(V)$ foi obtido pela soma dos volumes individuais $\left(v_{\mathrm{i}}\right)$, referentes as $(m)$ árvores medidas na unidade, e multiplicado pelo fator de proporcionalidade, da seguinte forma:

$$
\mathrm{V}=\left(\sum_{i=1}^{m} v_{i}\right)
$$

O volume comercial das árvores amostradas, nos métodos de Área Fixa, Prodan, Quadrantes e Bitterlich, foi calculado através das equações volumétricas desenvolvidas por IBDF/FATEC (1983), no Inventário das Florestas Nativas do Rio Grande do Sul, como segue:

a) Para espécies folhosas: 


$$
\log v=-3,92528+2,0425 \log d+0,6146 \log h
$$

b) Para Araucaria angustifolia:

$$
\log v=-4,2974+2,1842 \log d+0,6850 h
$$

Em que: $\quad v=$ volume individual comercial, $\mathrm{em} \mathrm{m}^{3}$ com casca;

d = diâmetro a altura do peito (DAP), em centímetros;

$\mathrm{h}=$ altura comercial, em metros.

\section{Método de Strand}

As unidades de Strand (linhas) foram instaladas a partir do início da primeira faixa, no sentido leste - oeste, estendendo-se uma trena para marcar o comprimento da unidade (L) de $15,7 \mathrm{~m}$, o qual era marcado no terreno por duas balizas.

Considerando-se que a altura dominante das árvores da floresta não ultrapassa $35 \mathrm{~m}$, de acordo ao inventário feito por IBDF/FATEC (1989), as linhas de Strand consideraram árvores situadas a uma distância máxima de 17,5 m. Por isso, cada linha ocupou duas faixas da parcela $(20 \mathrm{~m})$, para evitar que um mesmo indivíduo fosse incluído em duas linhas de Strand.

Uma vez marcada a linha, procedia-se a seleção das árvores situadas no seu lado esquerdo em duas etapas:

- Na primeira etapa, caminhando-se sobre a linha, selecionaram-se as árvores com critério probabilístico proporcional ao diâmetro, através do reslascópio de Bitterlich, usando-se o FAB (fator de área basal) igual a 4.

$\mathrm{Na}$ segunda etapa, caminhando-se novamente sobre a linha, selecionaram-se as árvores com critério probabilístico proporcional à altura, incluindo-se todas as árvores cuja distância até a linha era menor ou igual à metade da sua altura total.

Em qualquer das etapas, cada árvore selecionada foi identificada botanicamente e mediuse sua CAP (circunferências a altura do peito) através de uma trena com precisão de milímetro. Registrou-se também o tempo utilizado desde a instalação da linha até o final da medição da unidade.

As linhas foram instaladas de forma contínua, resultando 6 unidades em cada duas faixas ou 30 unidades de Strand na parcela permanente.

a) Estimativa da área basal por hectare expressão:

A área basal (G) foi obtida através da seleção proporcional ao diâmetro, pela seguinte

$$
G=\frac{\sqrt{F A B}}{10} \sum_{i=1}^{m} d_{i}
$$


Em que:

$d_{i}=$ diâmetro das arvores selecionadas, em centímetros;

$F A B=$ Fator de área basal.

b) Estimativa do número de árvores por hectare

O estimador do número de árvores por hectare ou densidade $(\mathrm{N})$ foi obtido através da equação desenvolvida por Péllico Netto \& Brena (1997), considerada apropriada para a o Método de Strand.

Neste caso, a estimação foi feita usando-se os dados obtidos pela seleção proporcional ao diâmetro $\left(\mathrm{d}_{\mathrm{i}}\right)$, através da expressão:

$$
N=\frac{200 \sqrt{F A B}}{L} \sum_{i=1}^{m}\left(\frac{1}{d_{i}}\right)
$$

Em que:

$m=$ número de árvores selecionadas;

$F A B=$ fator de área basal;

$L=$ comprimento da linha;

$d_{i}=$ DAP das árvores selecionadas.

Neste caso, os diâmetros foram medidos em centímetros.

c) Estimativa do volume por hectare

Para o cálculo do volume, usaram-se dois fatores de forma médios derivados das equações de volumes anteriores, uma para as araucárias e outro para as folhosas, cujos valores foram de 0,500 e 0,4244 respectivamente. Estes fatores de forma expressam o volume comercial a partir da altura total das árvores selecionadas. A equação usada para o cálculo do fator de forma foi a proposta por Finger (1992), como segue:

Em que:

$$
\int 1,3=\frac{\bar{v}}{v}
$$

$\int 1,3$ = fator de forma a $1,30 \mathrm{~m}$ do solo;

= volume da árvore média;

$\mathrm{v}=$ volume do cilindro.

A estimativa do volume por hectare (V) é obtida através da seleção proporcional à altura, pela seguinte expressão:

$$
V=\int \frac{1}{10} \sum_{i=1}^{m} d_{i}
$$

Em que: $\int=$ fator de forma médio do povoamento ou das espécies. 


\section{Método de Prodan}

A aplicação do método de Prodan foi feita distribuindo-se os pontos amostrais a cada $20 \mathrm{~m}$, a partir do cruzamento das linhas limites entre as faixas I e 2, e as subunidades I e 2, formando uma rede sistemática composta de 25 pontos.

Os pontos foram facilmente localizados uma vez que todas as interseções limites de faixas com subunidades, na parcela permanente, encontram-se marcadas com estacas.

Em cada ponto, selecionaram-se as seis árvores mais próximas do mesmo e mediu-se a distância radial da sexta árvore, utilizando-se uma trena com precisão de centímetros. Para cada árvore selecionada registrou-se a identificação da espécie, a medição da CAP, por meio de uma trena com precisão de milímetro, e a medição das alturas comercial e total com Blume-Leiss.

O tempo decorrido desde o início até a conclusão de cada ponto amostral de Prodan foi medido em minutos.

As equações utilizadas para as estimativas de área basal, número de árvores e volume comercial com casca por hectare, foram as seguintes:

a) Estimativa da área basal por hectare

A estimativa da área basal por hectare $(\mathrm{G})$ foi obtida através da seguinte expressão (PÉLLICO NETTO \& BRENA, 1997):

$$
G \frac{d_{1}^{2}+d_{2}^{2}+d_{3}^{2}+d_{4}^{2}+d_{5}^{2}+\frac{d_{6}^{2}}{2}}{R_{6}^{2}} \times 2.500
$$

Em que:

$d_{i}=$ diâmetro a altura do peito (DAP) de cada árvore da unidade;

$\mathrm{R}_{6}=$ raio da sexta árvore medida.

b) Estimativa do número de árvores por hectare

O número de árvores por hectare $(\mathrm{N})$ foi obtido pela seguinte expressão (PÉLLICO NETTO \& BRENA, 1997):

$$
N \frac{55.000}{\pi R_{6}^{2}}
$$

c) Estimativa do volume por hectare

A estimativa do volume por hectare $(\mathrm{V})$ foi obtida a partir do volume individual $\left(\mathrm{v}_{\mathrm{i}}\right)$ de cada uma das 6 árvores amostradas, através da seguinte expressão: 


$$
V=\frac{V 1+V 2+V 3+V 4+V 5+\frac{V 6}{2}}{\pi R_{6}^{2}} \times(10.000)
$$

Os volumes individuais foram calculados com as equações apresentadas no método de Área Fixa.

\section{Método dos Quadrantes}

O método dos Quadrantes foi aplicado sobre a parcela permanente usando-se os mesmos pontos amostrais definidos para o método de Prodan. $\mathrm{O}$ tempo foi medido desde o início até o final das medições. No ponto amostral o primeiro quadrante foi estabelecido como o noroeste, o segundo o sudoeste, o terceiro o sudeste e o quarto quadrante foi o nordeste. Em cada quadrante foi selecionada a árvore mais próxima do ponto amostral. As árvores selecionadas foram identificadas botanicamente, medidas as CAP e suas alturas total e comercial. A CAP e a distância de cada árvore selecionada ao ponto amostral foram medidas com uma trena com precisão de milímetros e as alturas com o Blume-Leiss.

As estimativas de número de árvores, área basal e volume por hectare foram obtidas seguintes expressões:

\section{a) Estimativa do número de árvores por hectare}

Para a estimativa do número de árvores por hectare, considerou-se a distância média total (Q) e as médias dos indivíduos de cada quadrante (), através das seguintes equações, desenvolvidas por Morisita apud Martins (1993):

$$
\left[\begin{array}{c}
\bar{Q}=\sqrt{\bar{M}} \\
\overline{Q_{1}}=0,50 \sqrt{\bar{M}} \\
\overline{Q_{2}}=0,81 \sqrt{\bar{M}} \\
\overline{Q_{3}}=1,12 \sqrt{\bar{M}} \\
\overline{Q_{4}}=1,57 \sqrt{\bar{M}}
\end{array}\right]
$$

Em que:

$$
\bar{Q}=\text { média das quatro distâncias; }
$$

$\bar{M}=\overline{Q_{2}}=$ área média de ocupação;

$\overline{Q_{l}}=$ distância radial média do ponto ao centro da árvore.

Assim, o número médio de árvores estimado por hectare $(\mathrm{N})$ foi calculado pela expressão (COTTAM \& CURTIS apud MARTINS, 1993):

$$
N=\frac{10.000}{\bar{M}}
$$

Ci. Fl., v. 1, n. 1, 1991 
b) Estimativa da área basal por hectare

A estimativa da área basal por hectare $(\mathrm{G})$ foi obtida a partir da somatória da área de seção transversal média $\left(\mathrm{g}_{\mathrm{i}}\right)$ das quatro árvores amostradas no ponto amostral, multiplicada pela densidade estimada, ou seja:

$$
G=\left(\frac{g_{1}+g_{2}+g_{3}+g_{4}}{4}\right) \times N
$$

Em que: $\mathrm{N}$ = número de árvores por hectare.

c) Estimativa do volume por hectare

Para a estimativa do volume comercial por hectare (V) usou-se a seguinte expressão:

$$
V=\left(\frac{v_{1}+v_{2}+v_{3}+v_{4}}{4}\right) \times N
$$

Em que:

$\mathrm{v}_{\mathrm{i}}=$ volume individual das árvores mais próximas em cada quadrante, em metros cúbicos.

\section{Método de Bitterlich}

A aplicação do método de Bitterlich sobre a parcela permanente utilizou os mesmos pontos amostrais definidos nos métodos de Prodan e dos Quadrantes.

Em cada ponto amostral, as árvores amostradas foram selecionadas por meio do relascópio de Bitterlich, usando-se o fator de área basal - FAB $=4$.

O tempo foi tomado desde a localização do ponto até a finalização das medições. No ponto amostral, realizou-se um giro de $360^{\circ}$ com o relascópio de Bitterlich e se fez a seleção das árvores em proporção a seu diâmetro, usando um fator de área basal de 4. Nas árvores selecionadas foram medidas as alturas totais e comerciais e as circunferências a altura do peito (CAP). As alturas foram medidas com altímetro Blume-Leiss e as circunferências com trena com precisão de milímetros.

As equações para a estimativa da área basal por hectare, número de árvores por hectare e volume comercial com casca por hectare, foram as seguintes:

\section{a) Estimativa da área basal por hectare}

A estimativa da área basal de cada ponto amostral $(\mathrm{G})$, expressa $\mathrm{em} \mathrm{m}^{2} / \mathrm{ha}$, foi determinada multiplicando-se o $F A B$ (fator de área basal) pelo número de árvores $(\mathrm{m})$ selecionadas (PÉLLICO NETTO \& BRENA, 1993; FINGER, 1992; LOETSCH et al., 1973; HUSCH et al., 1982), como segue:

$$
\mathrm{G}=m \cdot F A B
$$


Moscovich, F. A.; Brena, D. A.; Longhi, S. J.

b) Estimativa do número de árvores por hectare

A estimativa do número de árvores por hectare $(\mathrm{N})$ foi obtida através da seguinte equação:

$$
N=\sum_{i=1}^{m} N_{i}
$$

$\mathrm{O}$ número de árvores por hectare que cada árvore selecionada $\left(\mathrm{N}_{\mathrm{i}}\right)$ representa é dado pela seguinte expressão:

$$
N_{i}=\frac{F A B}{g_{i}}
$$

Em que:

$g_{i}=$ área transversal da i-ésima árvore medida no ponto amostral.

c) Estimativa do volume por hectare

A partir das equações volumétricas apresentadas no método de Área Fixa, calculou-se o volume individual de cada árvore incluída no ponto amostral $\left(\mathrm{V}_{\mathrm{i}}\right)$. Multiplicou-se o volume de cada árvore pelo respectivo número de árvores por hectare, obtendo-se o conversor do volume por hectare, correspondente a cada árvore amostrada (PÉLLICO NETTO \& BRENA, 1997).

$$
\mathrm{V}_{1}=N_{i} v_{i}
$$

$\mathrm{O}$ volume por hectare foi obtido, somando-se os estimadores individuais $\left(\mathrm{V}_{\mathrm{i}}\right)$ para as $(m)$ árvores inclinadas no ponto amostral, como segue:

$$
V=\sum_{i=1}^{m} V_{i}
$$

Em qualquer um dos métodos, os dados foram coletados como segue: CAP (circunferência a $1,30 \mathrm{~m}$ do nível do solo ou circunferência a altura do peito) de todas as árvores cuja CAP era maior ou igual a $30 \mathrm{~cm}$ e as alturas total e comercial, medidas com Blume-Leiss. A exceção foi o método de Strand que dispensava a medição das alturas das árvores, sendo necessária apenas a medição da CAP, tanto na seleção proporcional ao diâmetro como na seleção proporcional à altura das árvores.

Os dados coletados nas unidades de Área Fixa representam a enumeração total ou censo da parcela permanente, cujo processamento dos mesmos resultaram os parâmetros quantitativos: área basal, número de árvores e volume comercial por hectare, e os parâmetros qualitativos: número de espécies, gêneros e famílias botânicas. Esses parâmetros constituíram a base de comparação dos resultados obtidos em cada método.

Os parâmetros fitossociológicos foram calculados para todas as unidades amostrais de Área Fixa. Através da caracterização da população, foi feita a comparação da amostragem das espécies obtidas pelos métodos de Strand, Prodan, Quadrante e Bitterlich, em função da Densidade Relativa (DR). Os parâmetros fitossociológicos foram calculados pelo programa FITOPAC 2, desenvolvido pelo Dr. George J. Shepherd, do Instituto de Botânica da Universidade de Campinas (UNICAMP).

Realizou-se a análise da variância das estimativas dos parâmetros quantitativos, obtida nos

Ci. Fl., v. 1, n. 1, 1991 
métodos de Área Fixa, Strand, Prodan, Quadrantes e Bitterlich. Havendo diferenças significativas entre eles, foi aplicado o teste de comparação de médias de Tukey (STORCK \& LOPES, 1997 e FONSECA \& MARTINS, 1996).

A comparação de eficiência, na estimativa da área basal, número de árvores e volume comercial com casca por hectare, de todos os métodos, foi feita através do cálculo da eficiência relativa (ER), de acordo com os procedimentos recomendados por Mesavage \& Grosenbaugh apud Silva Mendes (1998). Para o cálculo da eficiência relativa dos diferentes parâmetros quantitativos estimados pelos distintos métodos, utilizou-se o critério proposto por Freese apud Péllico Netto \& Brena (1997), considerando os tempos médios de medição em cada um dos métodos e os coeficientes de variação obtidos nas estimativas dos parâmetros, como segue:

Em que:

$$
E R=\frac{1}{T \times C V^{2}}
$$

$\mathrm{ER}=$ Eficiência relativa;

$\mathrm{T}_{\mathrm{X}}=$ Tempo de medição;

$\mathrm{CV}=$ Coeficiente de variação.

\section{RESULTADOS E DISCUSSÃO}

De acordo com o levantamento feito na unidade amostral de um hectare, foram medidas 890 árvores com CAP maior ou igual a $30 \mathrm{~cm}$, das quais foram identificadas, para a vegetação floresta, 53 espécies, 42 gêneros, e 30 famílias botânicas, além das árvores mortas e não identificadas, que também fazem parte deste estudo.

De acordo com a análise fitossociológica fornecida pelo método de Área Fixa, observou-se que a espécie com maior densidade foi Araucaria angustifolia (Bert.) O. Ktze. (pinheiro-brasileiro), com 353 árvores/ha (39,66\% do total das árvores amostradas), seguindo em ordem decrescente: Ilex brevicuspis Berg. (caúna) com 59 árvores/ha (6,63\%); Blepharocalyx salicifolius (Kunth.) Berg. (murta) com 58 árvores/ha (6,52\%); Luehea divaricata Mart. (açoita-cavalo) com 46 árvores/ ha $(5,17 \%)$; Ilex paraguariensis A. St. Hil. (erva-mate) e Campomanesia xanthocarpa Berg. (guabirobeira) com 22 árvores/ha (2,47\%); e Banara parvifolia (A. Gray) Benth. (guaçatunga) e Sebastiania commersoniana (Baill.) Smith \& Downs (branquilho) com 19 árvores/ha (2,47\%) cada uma. Estas espécies totalizam um valor de importância (VI) de 187,35 e um valor de cobertura (VC) de 154,68. É uma Floresta Ombrófila Mista típica, na qual a Araucaria angustifolia é a espécie predominante do estrato superior da floresta.

$\mathrm{Na}$ Tabela 1 pode-se observar, de modo comparativo, a abrangência das principais espécies (em percentagem) nos diferentes métodos, tomando-se como parâmetro o método de Área Fixa. Constata-se que os mesmos têm uma distribuição semelhante, com uma grande percentagem de ocorrência de Araucaria angustifolia e uma pouca representação das outras espécies. Nesta tabela pode-se observar que todos os métodos (Prodan, Strand, Quadrantes e Bitterlich) amostraram as espécies mais representativas da fitossociologia desta floresta. Salienta-se que as espécies mais representativas também foram as mais densas nos distintos métodos empregados. 
TABELA 1: Densidade relativa das 9 espécies mais importantes encontradas nos métodos de amostragem em estudo.

TABLE 1: Relative density of the 9 most important species found in the sampling methods under study.

\begin{tabular}{lccccc}
\hline \multirow{2}{*}{$\begin{array}{c}\text { Espécies } \\
\text { (Nome Científico) }\end{array}$} & \multicolumn{5}{c}{ Métodos/Densidade Relativa (\%) } \\
\cline { 2 - 5 } & Área Fixa & Prodan & Bitterlich & Quadrantes & Strand \\
\hline Araucaria angustifolia & 39,66 & 36,67 & 72,71 & 42,00 & 51,53 \\
Ilex brevicuspis & 6,63 & 4,67 & 4,01 & 8,00 & 3,43 \\
Blepharocalyx salicifolius & 6,52 & 2,67 & 2,67 & 3,00 & 5,91 \\
Mortas não identificadas & 6,40 & 6,67 & 2,34 & 4,00 & 4,77 \\
Luehea divaricata & 5,17 & 6,00 & 2,01 & 4,00 & 4,20 \\
Campomanesia xanthocarpa & 2,47 & 2,00 & 0,33 & 1,00 & 1,72 \\
Ilex paraguariensis & 2,47 & 2,67 & 2,67 & 3,00 & 2,48 \\
Banara parvifolia & 2,13 & 5,33 & 0,33 & 4,00 & 1,33 \\
Sebastiania commersoniana & 2,13 & 0,67 & 0,00 & 1,00 & 1,91 \\
\hline
\end{tabular}

Na Tabela 2 podem ser observados os resultados das estimativas para volume comercial médio com casca por hectare, área basal média por hectare, número de árvores médio por hectare, tempos de medição médios, número de espécies médio, erro padrão e coeficiente de variação para todos os métodos empregados.

Os resultados das análises de variâncias do volume comercial com casca por hectare, área basal por hectare, número de árvores por hectare e abrangência de espécies, são apresentados na Tabela 3.

De acordo com a Tabela 3, verifica-se que não houve diferenças estatísticas significativas entre os métodos, com $1 \%$ de erro, na estimativa do volume comercial com casca, área basal e número de árvores por hectare.

De acordo com os valores estimados nos diferentes métodos de amostragem, para a estimativa de volume comercial com casca por hectare e, comparando-se estes resultados com os obtidos pelo método de Área Fixa, constata-se que o método dos Quadrantes e o método de Strand obtiveram uma superestimava do volume comercial, enquanto os métodos de Bitterlich e Prodan obtiveram uma subestimativa deste parâmetro. $\mathrm{O}$ método de Strand foi o que mais superestimou o volume (+32,26\%); o método dos Quadrantes obteve uma superestimativa de $+25,81 \%$.

O método de Bitterlich obteve a subestimativa do volume comercial (-8,78\%), e o método de Prodan uma subestimativa de $-27,14 \%$. Verifica-se, portanto, que o método de Bitterlich foi o que obteve a estimativa do volume comercial com casca mais próxima do valor paramétrico.

$\mathrm{Na}$ estimativa da área basal por hectare, o método dos Quadrantes foi o único que 
TABELA 2: Estimativas de volume, área basal, número de árvores, com respectivos coeficientes de variação e erro padrão (em porcentagem), tempo médio de medição de uma unidade e número de espécies amostradas em cada método.

TABLE 2: Volume estimates, basal area, number of trees, with respective coefficients of variation and standard error (in percentage), mean time of measurement of one unit and number of species sampled in each method

\begin{tabular}{cccccc}
\hline \multirow{2}{*}{ Parâmetros } & \multicolumn{5}{c}{ Métodos } \\
\cline { 2 - 5 } & Área Fixa & Strand & Prodan & Bitterlich & Quadrantes \\
\hline Vol.Com.(m $/$ ha) & 411,11 & 539,60 & 299,55 & 375,00 & 517,18 \\
\hline CV\% & 12,97 & 42,52 & 49,84 & 34,11 & 107,51 \\
S\% & 4,10 & 0,90 & 9,96 & 6,82 & 21,50 \\
Área Basal (m²/ha) & 53,16 & 48,30 & 46,58 & 48,00 & 63,65 \\
CV\% & 12,32 & 38,47 & 55,55 & 31,18 & 103,65 \\
S \% & 3,89 & 7,01 & 11,03 & 6,22 & 20,72 \\
N\%/ha & 889,20 & 878,00 & 811,56 & 983,80 & 1126 \\
CV\% & 9,03 & 51,80 & 40,68 & 45,93 & 78,03 \\
S\% & 2,85 & 5,45 & 8,13 & 9,18 & 15,60 \\
Tempo Médio & $4 \mathrm{~h} 45 \mathrm{~min} 59 \mathrm{~s}$ & $4 \min 40 \mathrm{~s}$ & $16 \mathrm{~min} 47 \mathrm{~s}$ & $33 \mathrm{~min} 17 \mathrm{~s}$ & $12 \mathrm{~min} 47 \mathrm{~s}$ \\
N. Espécies Médio & 20 & 4,9 & 3,92 & 3,52 & 3 \\
N. Espécies Total & 53 & 37 & 32 & 23 & 28 \\
\hline
\end{tabular}

superestimou este valor em relação ao parâmetro obtido pelo método de Área Fixa (+ 19,73\%). Os métodos de Prodan, Bitterlich e de Strand subestimaram a área basal por hectare em -12,38\%, $-9.14 \%$ respectivamente. Portanto, o método de Strand foi o que obteve a estimativa da área basal por hectare mais próxima do valor paramétrico.

$\mathrm{Na}$ estimativa do número de árvores por hectare constata-se que os métodos dos Quadrantes e de Bitterlich superestimaram o número de árvores por hectare em $+26,66 \% \mathrm{e}+19,57 \%$, respectivamente. Os métodos de Prodan e de Strand subestimaram este valor em $-8,77 \%$ e $-1,24 \%$ respectivamente. De acordo com estes resultados, o método de Strand foi o melhor estimador deste parâmetro.

Já para a amostragem de espécies, verificaram-se diferenças significativas, em nível de $1 \%$ de erro, na estimativa do número de espécies amostradas. Este resultado faz necessária a aplicação de um teste de comparação de médias para poder determinar quais são os métodos que não diferem significativamente do método de Área Fixa. Na Tabela 4 pode-se observar o teste de comparação de médias de Tukey em um nível de $1 \%$ de probabilidade de erro.

Através da Tabela 4, verifica-se que a maior média foi obtida pelo método de Área Fixa 
Moscovich, F. A.; Brena, D. A.; Longhi, S. J.

e a menor média pelo método dos Quadrantes. As médias obtidas pelos métodos de Bitterlich e de Prodan não diferem estatisticamente do método dos Quadrantes. Finalmente, verifica-se que entre as médias dos métodos de Strand, Prodan e Bitterlich não existem diferenças significativas, ou seja, cada uma das faixas do método de Área Fixa, abrange mais espécies que cada uma das unidades amostrais dos outros métodos, situação esperada devido à superfície maior de amostragem de uma faixa em relação aos pontos ou linhas dos outros métodos.

TABELA 3: Análise da variância para o volume comercial com casca por hectare $\left(\mathrm{m}^{3} /\right.$ ha), área basal por hectare $\left(\mathrm{m}^{2} / \mathrm{ha}\right)$, número de árvores por hectare $\mathrm{e}$ abrangência de espécies estimados pelos métodos de amostragem em estudo.

TABLE 3: Analysis of variance for the commercial volume with bark per hectare $\left(\mathrm{m}^{3}\right.$ / ha), basal area per hectare $\left(\mathrm{m}^{2} / \mathrm{ha}\right)$, number of trees per hectare and range of species estimated by the sampling methods under study.

\begin{tabular}{cccccc}
\hline \multirow{2}{*}{$\begin{array}{c}\text { Fonte de } \\
\text { Variação }\end{array}$} & GL & Volume (m/ha) & Área Basal (m²/ha) & N. árvores/ha & $\begin{array}{c}\text { Abrangência } \\
\text { de espécies }\end{array}$ \\
\cline { 3 - 6 } & & $262806,9^{1}$ & $1235,9^{1}$ & $366891,8^{1}$ & $606,2^{2}$ \\
\hline Método & 4 & 1239,1 & 1239,1 & 291767,1 & 2,9 \\
Erro & 105 & 51,61 & 51,61 & 941,35 & 5,29 \\
Média & & 68,20 & 68,20 & 57,38 & 32,10 \\
CV\% & & &
\end{tabular}

Em que: ${ }^{1}$ não significativo; ${ }^{2}$ significativo.

TABELA 4: Médias estimadas do número de espécies amostradas pelos diferentes métodos com classificação pelo teste de Tukey em $1 \%$.

TABLE 4: Estimated means of the number of species sampled by the different methods with at $1 \%$ Tukey test.

\begin{tabular}{ccccc}
\hline Métodos & \multicolumn{4}{c}{ Médias de espécies } \\
\hline Área Fixa & 20,00 & $\mathrm{a}$ & $\mathrm{b}$ & \\
Strand & 4,90 & & $\mathrm{~b}$ & $\mathrm{c}$ \\
Prodan & 3,92 & & $\mathrm{~b}$ & $\mathrm{c}$ \\
Bitterlich & 3,52 & & $\mathrm{c}$ \\
Quadrantes & 3,00 & & & \\
\hline
\end{tabular}

Em que: Métodos com medias não ligadas por mesma letra diferem pelo teste de Tukey em nível de $1 \%$ de probabilidade de erro. 
TABELA 5: Tempos médios, coeficiente de variação (cv) e eficiência relativa (ER) para cada método de amostragem e para os diferentes parâmetros estimados, volume comercial com casca $\left(\mathrm{m}^{3} / \mathrm{ha}\right)$, área basal $\left(\mathrm{m}^{2} / \mathrm{ha}\right)$ e número de árvores por hectare $\left(\mathrm{N}^{\circ} / \mathrm{ha}\right)$.

TABLE 5: Mean times, coefficient of variation (cv) and relative efficiency (ER) for each sampling method and for the different estimated parameters, commercial volume with bark $\left(\mathrm{m}^{3} / \mathrm{ha}\right)$, basal area $\left(\mathrm{m}^{2} / \mathrm{ha}\right)$ and number of trees per hectare $\left(\mathrm{N}^{\circ} / \mathrm{ha}\right)$.

\begin{tabular}{|c|c|c|c|c|c|c|c|}
\hline \multirow{2}{*}{ Métodos } & \multirow{2}{*}{$\begin{array}{l}\text { Tempo } \\
\text { Médio } \\
\text { (h) }\end{array}$} & \multicolumn{2}{|c|}{$\begin{array}{l}\text { Volume comercial }\left(\mathrm{m}^{3} /\right. \\
\mathrm{ha})\end{array}$} & \multicolumn{2}{|c|}{ Área basal (m²/ha) } & \multicolumn{2}{|c|}{ N./ha } \\
\hline & & $\mathrm{CV} \%$ & $\mathrm{ER} 1 / \mathrm{T}_{\mathrm{x}} \mathrm{CV}^{2}$ & $\mathrm{CV} \%$ & $\begin{array}{l}\mathrm{ER} \mathrm{1/} \\
\mathrm{T}_{\mathrm{x}} \mathrm{CV}^{2} \\
\end{array}$ & $\mathrm{CV} \%$ & $\begin{array}{l}\text { ER 1/ } \\
\mathrm{T}_{\mathrm{x}} \mathrm{CV}^{2} \\
\end{array}$ \\
\hline Área Fixa & 4,7663 & 12,9692 & 12,4736 & 12,3229 & 6,6879 & 9,0339 & 25,7080 \\
\hline Quadrantes & 0,2130 & 107,5127 & 4,0616 & 103,6501 & 4,3699 & 78,0301 & 7,7107 \\
\hline Prodan & 0,2797 & 49,8405 & 14,3927 & 55,5554 & 11,5839 & 40,6763 & 21,6085 \\
\hline Strand & 0,0778 & 42,5259 & 71,0744 & 38,4725 & 86,8400 & 51,7969 & 47,9085 \\
\hline Bitterlich & 0,5547 & 34,1145 & 15,4904 & 31,1805 & 18,5428 & 45,9326 & 8,5448 \\
\hline
\end{tabular}

Embora considerando o total de espécies amostradas pelos 25 pontos de amostragem de Prodan, Bitterlich e Quadrantes, e as 30 linhas de Strand, verifica-se que o método de Strand foi o que apresentou maior abrangência de espécies $(69,81 \%$ do total de espécies existentes na área em estudo), seguido pelo método de Prodan (60,38\%), dos Quadrantes (52,83\%) e de Bitterlich

Para o cálculo de eficiência relativa, utilizou-se o método proposto por Freese apud Pélllco Netto \& Brena (1997), para comparar métodos amostrais, incluindo o componente custo através dos tempos empregados na medição de cada uma das unidades amostrais. Na Tabela 5 observa-se que, em todos os casos, a maior eficiência ocorreu com o método de Strand. Este resultado está ligado à sua principal vantagem que é a não medição das alturas. Isto leva à realização de levantamentos muito rápidos, pois só é preciso a contagem das árvores selecionadas com proporcionalidade à altura e diâmetro, a identificação da espécie e a medição do diâmetro a altura do peito. O método dos Quadrantes sempre ocupou o último lugar em eficiência relativa, resultando ser o menos eficiente em sua aplicação em função dos custos e dos valores obtidos pelas suas estimativas.

Ainda, na Tabela 5, observa-se que os métodos de Prodan e de Bitterlich apresentaram boas estimativas, com boa eficiência relativa em relação à estimativa dos diferentes parâmetros considerados. Com respeito à eficiência relativa, o método de Prodan ocupou sempre o terceiro lugar, enquanto que o método de Bitterlich ocupou o segundo nas estimativas de volume e área basal e o quarto lugar na estimativa do número de árvores por hectare. 
Moscovich, F. A.; Brena, D. A.; Longhi, S. J.

\section{CONCLUSÕES}

Foram encontradas na área amostrada da Floresta Ombrófila Mista da Floresta Nacional de São Francisco de Paula, 53 espécies, distribuídas em 42 géneros e 30 famílias botânicas, indicando grande diversidade florística. As espécies Araucaria angustifolia, Ilex brevicuspis, Blepharocalyx salicifolius, Luehea divaricata, Ilex paraguariensis, Campomanesia xanthocarpa, Banara parvifolia e Sebastiania commersoniana foram as mais características da área analisada.

Os métodos em estudo não apresentaram diferença significativas em nível de $1 \%$ de erro para as estimativas de volume comercial com casca, área basal e número de árvores por hectare. $\mathrm{O}$ método de Bitterlich foi o que mais se aproximou ao valor real do volume por hectare; o método de Strand foi o que mais se aproximou do valor real da área basal por hectare e do número de árvores por hectare.

Para os resultados de abrangência de espécies foi encontrada diferença significativa entre as médias dos métodos, em nível de $1 \%$ de erro. O método de Área Fixa foi o que apresentou a maior média, diferindo significativamente dos resultados dos outros métodos. Porém, entre os métodos de Strand, Prodan, Quadrantes e Bitterlich não foi encontrada diferença significativa. Entre estes métodos, comparados com o método de Área Fixa (parâmetro), o método de Strand teve a abrangência de maior número de espécies (37 espécies), seguindo em ordem decrescente os métodos de Prodan (32 espécies), Quadrantes (28 espécies) e Bitterlich (23 espécies).

O método de Strand foi o método que obteve a maior eficiência relativa na estimativa do todos os parâmetros. Em contraposição, o método dos Quadrantes foi o que apresentou a menor eficiência relativa em todos os parâmetros.

De acordo com este estudo e dependendo do objetivo do inventário, o método de Strand pode ser utilizado para a realização de inventário florestal na Floresta Ombrófila Mista da Floresta Nacional de São Francisco de Paula, uma vez que apresentou o melhor conjunto de resultados, tanto quantitativos (volume comercial, área basal, número de árvores por hectare) como qualitativo (abrangência de espécies), em menor tempo.

\section{REFERÊNCIAS BIBLIOGRÁFICAS}

BRENA, D.A. Notas da aula. UNIVERSIDADE FEDERAL DE SANTA MARIA. 1996.

BRASIL. Ministério da Agricultura. Departamento Nacional de Pesquisa Agropecuária.

Levantamento de reconhecimento dos solos do Estado do Rio Grande do Sul. Recife: 1973. 431 p. (Boletim Técnico, n.30).

FINGER, C.A.G. Fundamentos de biometria florestal. Santa Maria: UFSM CEPEF/FATEC, 1993. $269 \mathrm{p}$.

FONSECA, J.S.; MARTINS, G.A. Curso de estatística. 6a ed. São Paulo: Ed. Atlas S.A., 6a ed., 1996. $320 \mathrm{p}$.

FONTANA, C.S. História natural de Heteroxolmis dominicana (VIEILLOT, 1823) (AVES TYRANNIDAE) com ênfase na relação ecológica com Xanthopsar flavus (GMELIN, 1788) (AVES ICTERIDAE), no nordeste do Rio Grande do Sul. Porto Alegre: 1994. 120 p. Dissertação 
(Mestrado). Instituto de Biociências, PONTIFÍCIA UNIVERSIDADE CATÓLICA HUECK, K. As florestas da América do sul. São Paulo: Ed. Polígono, 1972. 466 p. HUSCH, F.; MILLER, C.I.; BEERS, T. W.; Forest mensuration. 3 ed. New York: John Wiley \& Sons, 19822. 402p.

IBDF/FATEC Inventario florestal nacional: Florestas Nativas do Rio Grande do Sul. Brasilia1: Edite Gráfica Brasiliana Ltda., 1983, 345 p.

IBDF/FATEC: Plano de para a Floresta Nacional de de Paula - RS. Santa Maria: Ministério Interior. Instituto do Brasileiro do Médio Ambiente e Recursos Naturais Renováveis, 1989. 217p.

KLEIN, R.M. O aspecto do Pinheiro Brasileiro. Sellowia, Itajaí, v.12, n.12, p.17-48, 1960.

LINDMAN, C.A.M.; FERRI, M.G. A vegetação do Rio Grande do sul. Belo Horizonte: Itatiaia, 1974. $377 \mathrm{p}$.

LOETSCH, F.; ZOHRER, F.; HALLER, K.E. Forest inventory. 2 ed. Munich: BLV Vellagsgesellschaft, 1973. 469p.

MARTINS, F.R. Estrutura de una Floresta Mesófila. Campinas: UNICAMP. 1991.246 p.

PÉLLICO NETTO, S., BRENA, D.A. Inventário florestal. Curitiba: Universidade Federal do Paraná - Universidade Federal de Santa Maria, 1997.316 p.

PRODAN, M. Forest biometrics. Oxford: Pergamon Press, 1968.447 p.

RAMBO, Pe. R. A fisionomia do Rio Grande do Sul. Porto Alegre: Livraria Selbach, 1956, 456p.

REITZ, R.; KLEIN, R.M. Flora ilustrada catarinense: Araucariáceas, Itajaí: Herbário Barbosa Rodrigues. $1966.63 \mathrm{p}$.

ROBLES, C. Estadística. Santiago del Estero: Facultad de Ciencias Forestales. Universidad Nacional de Santiago del Estero, 1978.285 p.

SILVA MENDES, I.M. da. Aplicação do método de amostragem de Strand para estimação da densidade na regeneração natural de espécies arbóreas e arbustivas tropicais na Amazônia Ocidental. Curitiba: Universidade Federal do Paraná, 1998. 51 p. Dissertação (Niestrado em Ciências Florestais). Setor de Ciências Agrárias, Universidade Federal do Paraná.

SOUZA, A.L. et al. Comparação de tipos de amostragem, com parcelas circulares de área fixa e variável, em povoamentos de Eucalvptus grandis, de origem híbrida, cultivados na região de Bom Despacho, Minas Gerais. Revista Arvore, Viçosa, v.5, n.1, p.43-55, 1981.

STORCK, L.; LOPES, S.J. Experimentação II. Santa Maria: UFSM, Centro de Ciências Rurais, Departamento de Fitotecnia, 1997. 197p.

VELOSO, H.P.; GÓES FILHO, L. Fotogeografia Brasileira: Classificação fisonômica-ecológica da vegetação neotropical, In: PROJETO RADAMBRASIL 1982, Salvador. 85 p. (Bol. Técnico Série vegetação, 1). 